\title{
O USO DA MICROBIOLOGIA COMO FERRAMENTA PARA A CONSERVAÇÃO DE AVES AMEAÇADAS: DADOS PRELIMINARES PARA O PAPAGAIO-DE-CARA-ROXA, Amazona brasiliensis (Aves: Psittacidae) NO PARANÁ
}

\author{
Patricia Pereira Serafini \\ Rafael Meurer ${ }^{2}$ \\ Sonia Maria Biesdorf ${ }^{3}$ \\ Elenise Angelotti Bastos Sipinski ${ }^{4}$
}

SERAFINI, P. P.; MEURER, R.; BIESDORF, S. M.; SIPINSKI, E. A. B. O uso da microbiologia como ferramenta para a conservação de aves ameaçadas: dados preliminares para o papagaio-de-cara-roxa, Amazona brasiliensis (Aves: Psittacidae) no Paraná. Arq. Ciênc. Vet. Zool. UNIPAR, Umuarama, v. 18, n. 1, p. 65-69, jan./mar. 2015.

RESUMO: Amazona brasiliensis, também conhecido popularmente como papagaio-de-cara-roxa, é uma espécie de Psittacidae endêmica da Mata Atlântica distribuída entre o litoral sul de São Paulo e o litoral Norte de Santa Catarina. Este estudo foi concentrado no estado do Paraná, visando uma prévia caracterização do perfil sanitário natural em filhotes da população por meio de análises microbiológicas. O estudo epidemiológico de uma espécie ameaçada inicia-se com a determinação dos agentes infecciosos comuns na população, que provavelmente co-evoluíram com a espécie e representam baixo risco aos espécimes de vida livre. Do total de colônias isoladas quase $75 \%$ foram positivas para a família Enterobacteriaceae. Normalmente, a microbiota entérica de psitacídeos é composta por micro-organismos Gram-positivos, porém a alta porcentagem de Gram-negativas isoladas pode ser explicada pela diferença entre a composição microbiana de adultos e filhotes. Seria interessante um estudo mais detalhado para uma eventual comparação entre possíveis sinais clínicos e micro-organismos presentes em cada indivíduo amostrado.

PALAVRAS-CHAVE: Microbiologia. Conservação. Amazona brasiliensis. Litoral do Paraná.

\section{USE OF MICROBIOLOGY AS A TOOL FOR THE PRESERVATION OF THREATENED BIRD SPECIES: PRELIMINARY DATA FOR THE RED-TAILED AMAZON, Amazona brasiliensis (Birds: Psittacidae) IN PARANÁ}

ABSTRACT: Amazona brasiliensis, also popularly known as Red-tailed Amazon, is an endemic species to the Atlantic Forest. This Psittacidae range goes from the south coast of São Paulo state to the northern coast of Santa Catarina state. This study focuses on the population in the state of Paraná, aiming to perform a preliminary characterization of the prevalence of natural pathogens in nestlings through microbiological analyses. The epidemiological study of an endangered species begins with the determination of common infectious agents within the population, which probably co-evolved with the species and represent a low risk to free-living specimens. Almost $75 \%$ of the colonies isolated were positive for Enterobacteriaceae. Usually, the enteric microbiota of psittacidae consists of Gram-positive microorganisms, but the high percentage of isolated Gram-negative bacteria can be explained by differences between the microbial composition of adults and nestlings. It would be interesting to further develop this study into a more detailed comparison between possible clinical signs and microorganisms present in each individual sampled.

KEYWORDS: Microbiology. Conservation. Amazona brasiliensis. Coast of the State of Paraná.

\section{EL USO DE LA MICROBIOLOGÍA COMO HERRAMIENTA PARA LA CONSERVACIÓN DE AVES AMENAZADAS: DATOS PRELIMINARES PARA EL LORO DE CARA PÚRPURA, Amazona brasiliensis (Aves: Psittacidae) EN PARANÁ}

RESUMEN: Amazona brasiliensis, popularmente conocido como el loro de cara púrpura, es una especie endémica de Psittacidae de la Mata Atlántica distribuido entre la costa sur de São Paulo y la costa norte de Santa Catarina. Este estudio se concentra en el estado de Paraná, buscando caracterizar preliminarmente el perfil sanitario natural en crías de la población, por medio del análisis microbiológico. El estudio epidemiológico de una especie amenazada comienza con la determinación de agentes infecciosos comunes en la población, que probablemente ha evolucionado con la especie y representan un riesgo bajo para especímenes de vida libre. Del total de colonias aisladas casi el 75\% fueron positivos para Enterobacteriaceae. Normalmente, la microbiota entérica de loros se compone de microorganismos Gram positivos, pero el alto porcentaje de aislados

DOI: https://doi.org/10.25110/arqvet.v18i1.2015.5371

${ }^{1}$ Analista Ambiental CEMAVE/Instituto Chico Mendes de Conservação da Biodiversidade, Mestre em Ciências Biológicas, Universidade Estadual de Londrina - UEL, Londrina - PR. patricia.serafini@icmbio.gov.br. ESEC Carijós/ICMBio. Base Avançada do CEMAVE em Santa Catarina. Rodovia Maurício Sirotski Sobrinho s/n km2 SC402, CEP:88.053-700. Bairro Jurerê. Florianópolis/SC.

${ }^{2}$ Graduando em Ciências Biológicas, Universidade Federal de Santa Catarina - UFSC, Florianópolis - SC.

${ }^{3}$ Servidora da Centro de Diagnóstico Marcos Enrietti, Curitiba -PR, Mestre em Ciências Veterinárias, Universidade Federal do Paraná -UFPR, Curitiba PR.

${ }^{4}$ Coordenadora do Projeto de Conservação do Papagaio-de-cara-roxa, Mestre em Conservação da Natureza na Engenharia Florestal, Universidade Federal do Paraná - UFPR, Curitiba - PR. 
Gram negativas se puede explicar por la diferencia entre la composición microbiana de adultos y pichones. Sería interesante un estudio más detallado para una posible comparación entre los síntomas clínicos y los microorganismos presentes en cada individuo muestreado.

PALABRAS CLAVE: Microbiología. Conservación. Amazona brasiliensis. Costa de Paraná.

\section{Introdução}

O Papagaio-de-cara-roxa, Amazona brasiliensis [Linnaeus, 1758] é uma espécie endêmica de ave brasileira pertencente à ordem dos Psitaciformes e à família Psittacidae que inicialmente tinha sua distribuição em uma faixa contínua de $3.075 \mathrm{~km}^{2}$ entre o litoral sul de São Paulo e o litoral norte de Santa Catarina (DIEFENBACH; GOLDHAMMER, 1986; SCHERER-NETO, 1989; SIPINSKI et al. 2014). Ameaças comuns à maioria das espécies da fauna tais como fragmentação e degradação ambiental, introdução de espécies exóticas, poluição e mudanças climáticas são compartilhadas por esta espécie e podem facilitar ou permitir a transmissão de doenças infecciosas (HEARD et al., 2013).

O estudo epidemiológico de uma espécie ameaçada de extinção inicia-se com a determinação dos agentes infecciosos comuns nas populações naturais, os quais provavelmente co-evoluíram com a espécie e representam baixo risco de epidemias (AGUIRRE et al., 2012). A invasão de espécies exóticas, incrementada pela globalização e pelo aquecimento global, permite que agentes infecciosos exóticos se disseminem rapidamente em populações nativas suscetíveis, estas por não terem evoluído paralelamente com estes agentes, não possuem defesas naturais (ALTIZER et al. 2013).

Hoje a faixa contínua de ambiente para o papagaio-de-cara-roxa não existe mais, devido ao grande número de habitats fragmentados, tanto para ocupação antrópica como para atividades pecuárias, agricultura e outras atividades econômicas (SIPINSKI et al. 2014). São conhecidos poucos efeitos das doenças infecciosas sobre as populações naturais de aves (AGUIRRE et al., 2012), porém diversos autores concordam que existe uma premente necessidade de integrar as teorias e experiências práticas nos campos da ecologia, demografia, taxonomia e genética ao da epidemiologia da vida selvagem, a fim de desenvolver abordagens práticas para a prevenção da extinção de espécies (SOULÉ, 1996; LAFFERTY; GERBER, 2002).

No ambiente natural, o efeito de uma doença infecciosa em uma população hospedeira não aumenta linearmente com seus efeitos patogênicos em hospedeiros individuais. Uma doença com alta taxa de letalidade acaba por extinguir seu hospedeiro rapidamente impedindo sua disseminação na comunidade. Os efeitos mais severos esperados na densidade populacional ocorrem envolvendo doenças de patogenicidade intermediária (AGUIRRE et al., 2012).

A disseminação de um agente infeccioso transmitido diretamente entre indivíduos aumenta proporcionalmente à densidade de hospedeiros suscetíveis, ou seja, a intensidade e a prevalência das doenças tendem a aumentar com a densidade populacional de seus hospedeiros (BOWMAN, 1999; XENOULIS et al. 2010). Para a maioria das doenças hospedeiro-específicas, populações raras ou ameaçadas pela redução populacional drástica estariam, portanto, menos suscetíveis à invasão por agentes infecciosos. Esta teoria da transmissão densidade-dependente prediz que doenças infecciosas dificilmente serão a causa única responsável pela ex- tinção de espécies (LAFFERTY; GERBER, 2002).

Uma variável importante nos modelos de transmissão de patógenos seria a presença de comportamento social gregário apresentado por diversas espécies animais, como a maioria dos psitacídeos (SICK, 1997; AGUIRRE et al., 2012). O modelo do estudo aqui apresentado, o papagaio-de-cara-roxa (Amazona brasiliensis), pode agregar-se em números superiores a mil indivíduos em alguns dormitórios com área reduzida (SIPINSKI et al. 2014; CARRILLO et al., 2002; SCHERER-NETO, 1989). Este comportamento gregário aumenta a possibilidade de contato entre indivíduos, mesmo quando os números populacionais são reduzidos, favorecendo a transmissão de doenças.

Os efeitos da fragmentação também podem contribuir indiretamente para a disseminação de doenças, antes raras. Quando a área natural disponível para as espécies é reduzida, muitos animais tendem a concentrar-se em remanescentes florestais (SERAFINI, 2003). Estas altas densidades populacionais artificiais podem colocar populações sob risco de epidemias (LAFFERTY; GERBER, 2002). A aglomeração de indivíduos pode ser ainda mais grave quando diminui os recursos naturais (e.g. alimento) que poderiam contribuir para a resistência dos indivíduos às doenças (BECK; LEVANDER, 2000). Por outro lado, ambientes fragmentados podem oferecer condições favoráveis para a proliferação de vetores e hospedeiros intermediários, além de aumentar o contato entre populações naturais com animais domésticos e de produção, aumentando dessa forma a prevalência de agentes infecciosos causadores de doenças (DEEM et al. 2005; SERAFINI et al., 2003; LAFFERTY; KURIS, 1999).

Além disso, o incremento da interface entre animais domésticos e animais selvagens deve ser estudado cuidadosamente, pois a transmissão de doenças pode ocorrer nas duas direções (KOHL 2012). Doenças provenientes de criações de perus domésticos ocasionaram a extinção de Tympanuchus cupido cupido (Tretaz-das-pradarias) (SIMBERLOFF, 1986). A invasão de espécies exóticas, incrementada pela globalização, permite que agentes infecciosos exóticos se disseminem rapidamente em populações nativas suscetíveis, estas por não terem co-evoluído com estes agentes, não possuem defesas naturais (COOPER, 1993).

Este trabalho foi realizado tendo como meta, uma prévia caracterização natural das bactérias isoladas em ninhegos de Amazona brasiliensis (L. 1758) na Área de Proteção Ambiental (APA) de Guaraqueçaba e no Parque Nacional de Superagui por meio da realização de análises microbiológicas.

\section{Análises, Resultados e Conclusões}

Os estudos foram concentrados nas ilhas: Rasa, Gamelas e Grande, pertencentes à Área de Proteção Ambiental de Guaraqueçaba, além da ilha das Peças, pertencente ao Parque Nacional do Superagui.

Neste trabalho foram utilizadas 19 amostras de fi- 
lhotes de Amazona brasiliensis na estação reprodutiva 20042005 entre 21 e 49 dias de vida. Cada amostra foi obtida por meio da coleta do conteúdo da cloaca com swabs bacteriológicos estéreis de haste plástica durante os monitoramentos dos ninhos na estação reprodutiva, que se inicia em setembro e termina em março, e são realizados anualmente pela equipe da SPVS (Sociedade de Pesquisa em Vida Selvagem e Educação Ambiental). $\mathrm{O}$ acesso aos ninhos se deu por meio de técnicas de escalada vertical. Swabs amostrados da cloaca de cada filhote de papagaio eram imediatamente armazenados em meio de transporte Stuart, que carece de uma fonte de nitrogênio para que os micro-organismos não se reproduzam, e permitiram que os mesmos permaneçam viáveis até a chegada ao Laboratório de Bacteriologia do Centro Diagnóstico Marcos Enrietti, CDME-SEAB (Curitiba-PR), onde todas as amostras foram semeadas em placas de petri em meio de cultura contendo Agar nutriente e posteriormente processadas.

Os resultados obtidos por meio da análise microbiológica dos cultivos das amostras cloacais de ninhegos de Amazona brasiliensis estão dispostos na Tabela 1. As amostras foram positivas em 84,21 \% para Escherichia. coli e das 47 culturas cultivadas de micro-organismos a partir de amostras cloacais , 74,46 \% foram positivas para membros da família Enterobacteriaceae.

Tabela 1: Número de amostras positivas para o micro-organismo (N. A. P. M.)

\begin{tabular}{lll}
\hline \multicolumn{1}{c}{ Família } & \multicolumn{1}{c}{ Espécies } & N. A. P. M. \\
\hline Cryptococcaceae & Candida spp. & 04 \\
\hline Enterobacteriaceae & $\begin{array}{l}\text { Citrobacter } \\
\text { amalonaticus }\end{array}$ & 01 \\
\hline Enterobacteriaceae & Citrobacter diversus & 03 \\
\hline Enterobacteriaceae & $\begin{array}{l}\text { Enterobacter } \\
\text { aerogenes }\end{array}$ & 01 \\
\hline Enterobacteriaceae & Pantoea aglomerans & 03 \\
\hline Enterobacteriaceae & Enterobacter cloacae & 02 \\
\hline Enterobacteriaceae & Enterobacter spp. & 02 \\
\hline Enterobacteriaceae & Escherichia coli & 16 \\
\hline Enterobacteriaceae & Proteus vulgaris & 05 \\
\hline Enterobacteriaceae & Providencia sp. & 02 \\
\hline Pseudomonadaceae & Pseudomonas spp. & 06 \\
\hline Staphylococcaceae & Staphylococcus sp. & 01 \\
\hline Streptomycetaceae & Streptomyces sp. & 01 \\
\hline
\end{tabular}

A microbiota intestinal de Psittaciformes é constituída principalmente por bactérias Gram-positivas, podendo aqui destacar os principais gêneros: Lactobacillus spp., $B a-$ cillus spp., Corynebacterium spp., Streptomyces spp., Gaffkya spp., Aerococcus spp., Micrococcus spp., Staphylococcus spp., e Streptococcus spp. não hemolíticos (BANGERT et al., 1988; FLAMMER; DREWES, 1988; MARIETTO-GONÇALVES et al., 2010; ALLEGRETTI et al., 2014). Trabulsi (1996) não considera saudável a presença de bactérias Gram-Negativas em um indivíduo, assim como Hoefer (1997) e Mattes et al. (2005) sugerem que Escherichia coli não é um componente natural da microbiota entérica de psitacídeos, portanto sua presença estaria associada com a ocor- rência de alguma enfermidade ou desequilíbrio (KNÖBL, T.; MENÃO, M. 2010). No trabalho de Saidenberg (2008), o autor obteve 40 amostras de filhotes de psitacídeos em vida livre e todas foram positivas para a família Enterobacteriaceae, sendo Escherichia coli a espécie mais abundante constando em 62,5\% das amostras (KNÖBL et al., 2011). Em trabalho similar, Bowman e Jacobson (1980) trabalharam com oito espécies de psitacídeos adultos clinicamente saudáveis, relatando uma baixa porcentagem de isolamento de bactérias Gram-negativas, sendo E. coli e Enterobacter spp. isoladas com maior frequência.

A composição da microbiota de filhotes e adultos de psitacídeos em vida livre é diferente devido às condições de higiene dos ninhos naturais, pois ocorre um acúmulo de fezes e matéria orgânica além da possível utilização prévia dos ninhos por outras espécies com a microbiota entérica divergente a de psitacídeos (e.g. e ranfastídeos) (VAN DONGEN et al., 2013; SAIDENBERG, 2008). Do total de amostras cloacais de papagaio-de-cara-roxa, $21 \%$ foi positivo para Candida spp., uma levedura que possui distribuição mundial e em pequenos números faz parte da microbiota intestinal normal (QUEIROZ, 2008), porém quando associada com a enfermidade, geralmente é o agente secundário ou terciário da infecção (ROSSKOPF; WOERPEL, 1996). Staphylococcus spp. foi positivo em 5,26\% das amostras de papagaio-de-cara-roxa, esta bactéria Gram-positiva poder ser encontrado como agente primário de infecções endógenas, são normalmente relatados como agentes oportunistas em doenças respiratórias que evoluem para um quadro septicêmico (caracterizada por sintomas clínicos inespecíficos, incluindo letargia, anorexia, penas eriçadas e morte súbita), apesar da maioria dos membros do gênero Staphylococcus serem considerados como parte natural da microbiota (QUEIROZ, 2008).

Pseudomonas spp. foi identificado em 31,57\% das amostras de papagaio-de-cara-roxa, esta, é uma bactéria Gram-negativa amplamente distribuída no mundo e é comumente encontrada em culturas com amostra de coana e cloaca de aves (QUEIROZ, 2008). Entretanto, quando é detectado fora do trato gastrointestinal ou da região da coana/orofaringe pode se tornar um perigoso agente patogênico (BROWN, 2000; DEEM et al., 2005; CUBAS et al., 2006). O único representante da família Streptomycetaceae, Streptomyces $s p$. foi isolado em 5,26\% sendo considerado como parte natural da microbiota intestinal (BANGERT et al., 1988; FLAMMER; DREWES, 1988).

Em aspectos gerais pode-se concluir que os 19 juvenis de Amazona brasiliensis amostrados neste trabalho estavam com um perfil sanitário dentro das normalidades, partindo do pressuposto que bactérias Gram-negativas em filhotes de Psitacídeos podem ser um caráter normal. Recomendamos um estudo mais detalhado para uma eventual comparação entre possíveis sinais clínicos e microorganismos presentes em cada indivíduo amostrado. Assim como, a continuidade do trabalho ampliando o número de indivíduos amostrados em diferentes anos, estações e condições de ninho.

Apenas com o conhecimento do que é natural para cada espécie, obtêm-se ferramentas para a avaliação de desequilíbrios na prevalência ou intensidade de parasitismo natural ou até mesmo da presença de agentes infecciosos introduzidos, potenciais causadores de epizootias. 


\section{Referências}

AGUIRRE, A.; OSTFELD, R.; DASZAK, P. New directions in conservation medicine: applied cases of ecological health. New York: Oxford University, 2014. 639 p.

ALLEGRETTI, L. et al. Isolation and molecular identification of lactic acid bacteria and Bifidobacterium spp. From faeces of the blue-fronted Amazon parrot in Brazil. Benef Microbes, v. 5, n. 4, p. 497-503, 2014.

ALTIZER, S. et al. Climate change and infectious diseases: from evidence to a predictive framework. Science, v. 341, p. 514-519, 2013.

BANGERT, R. L. et al. A survey of aerobic bacteria and fungi in the feces of healthy psittacine birds. Avian Diseases, v. 32, n. 1, p. 46-52, 1988.

BECK, M. A.; LEVANDER, O. A. Host nutritional status and its effect on a viral pathogen. Journal of Infectious Diseases, v. 182, p. 593-596, 2000.

BOWMAN, T. A.; JACOBSON, E. R. Cloacal flora of clinically normal captive psittacine birds. The Journal of Zoo Animal Medicine, v. 11, n. 3, p. 81-85, 1980.

BOWMAN, D. D. Georgis' parasitology for veterinarians. 7. ed. Philadelphia: Saunders, 1999. 414 p.

BROWN, N. H. H. Psittacine birds. In: TULLY JUNIOR, T. N.; LAWTON, M. P. C.; DORRESTEIN, G. M. Avian medicine. Oxford: Reed Educational and Professional Publishing, 2000.

CARRILLO, A. et al. Conservação do papagaio-de-cararoxa (Amazona brasiliensis) no Estado do Paraná. In: GALETTI, M.; PIZO, M. A. (Eds). Ecologia e conservação de psitacídeos no Brasil. Belo Horizonte: Melopsittacus Publicações Científicas, 2002. p. 193-213.

COOPER, J. E. Historical survey of disease in birds. Journal of Zoo and Wildlife Medicine, v. 24, p. 256-264, 1993.

CUBAS, Z. S.; SILVA, J. C. R.; CATÃO-DIAS, J. L. Tratado de animais selvagens - medicina veterinária. São Paulo: Roca, 2006.

DEEM, S. L. et al. Health evaluation of free-ranging and captive blue-fronted Amazon parrots (Amazona aestiva) in the Gran chaco, Bolivia. Journal of Zoo and Wildlife Medicine, v. 36, n. 4, p. 598-605, 2005.

DIEFENBACH, K. H.; GOLDHAMMER, S. P. Biologie und okologie der rotschwanzamazone Amazona brasiliensis. Trochilus, v. 7, p. 72-78, 1986.

FLAMMER, K.; DREWES, L. A. Species-related differences in the incidence of gram-negative bacteria isolated from the cloaca of clinically normal psittacine birds. Avian Diseases, v. 32, n. 32, p. 79-83, 1988.

HEARD, M. J. et al. The threat of disease increases as species move toward extinction. Conservation Biology, p. $1-11,2013$

HOEFER, H. L. Diseases of the gastrointestinal tract. In: ALTMAN, R. et al. Avian medicine and surgery. (Eds.). Philadelphia: Saunders. 1997. p. 419-453.

KNÖBL, T.; MENÃO, M. Escherichia coli enteropatogênica (EPEC) isoladas de psitacídeos. FIEP Bulletin, v. 80, p. 839-841, 2010.

KNÖBL, T. et al. Serogroups and virulence genes of Escherichia coli isolated from psittacine birds. Pesquisa Veterinária Brasileira, v. 31, n. 10, p. 916-921, 2011.

KOHL, K. D. Diversity and function of the avian gut microbiota. Journal of Comparative Physiology B, v. 182, p. 591-602, 2012.

LAFFERTY, K. D.; GERBER L. R. Good medicine for conservation biology: the intersection of epidemiology and conservation theory. Conservation Biology, v. 16, n. 3, p. 593-604, 2002.

LAFFERTY, K. D.; KURIS A. M. How environmental stress affects the impacts of parasites. Limnology and Oceanography, v. 44, p. 564-590, 1999.

MARIETTO-GONÇALVES, G. A. et al. Detecção de Escherichia coli e Salmonella sp. em microbiota intestinal de psittaciformes em fase de reabilitação para soltura. Brazilian Journal of Veterinary Research and Animal Science, v. 47, p. 185-189, 2010.

MATTES, B. R. et al. Influência da biossegurança na colonização intestinal por Escherichia coli em psitacídeos. Arquivo Instituto Biológico, v. 72, n. 2, p. 13-16, 2005.

QUEIROZ, B. D. Principais doenças respiratórias que acometem psitacídeos e passeriformes criados como animais de estimação. Rio de Janeiro, 2008. 78 f. Monografia (Trabalho de Conclusão de Curso) Universidade Castelo Branco, São Paulo, 2008.

ROSSKOPF, W. J.; WOERPEL, R. W. Diseases of cage and aviary birds Philadelphia. USA: A Lea \& Febiger Book, 1996.

SAIDENBERG, A. B. S. Detecção de fatores de virulência de Escherichia coli isoladas de psitacídeos com diferentes manifestações clínicas. 2008. 91 f. Dissertação (Mestrado em Medicina Veterinária) - Universidade de São Paulo, São Paulo, 2008.

SCHERER-NETO, P. Contribuição à biologia do papagaio-de-cara-roxa, Amazona brasiliensis (LINNAEUS, 1758) (Psittacidae, aves). 1989. $170 \mathrm{f}$. 
Dissertação (Mestrado em Zoologia) - Universidade Federal do Paraná, Curitiba, 1989.

SERAFINI, P. P. et al. First Report of Ornithonyssus sylviarum (Acari: Macronyssidae) on Black Vulture (Coragyps atratus) nestlings from Brazil. Revista Brasileira de Parasitologia Veterinária, v. 12, n. 2, p. $92-$ 93, 2003.

SERAFINI, P. P. Populações de Psittacidae no Parque Estadual Mata dos Godoy, Fragmento de Floresta Atlântica no norte do Estado do Paraná. 2003. 74 f. Dissertação (Mestrado em Ciências Biológicas) Universidade Estadual de Londrina, Londrina, 2003.

SICK, H. Ornitologia brasileira. São Paulo: Nova Fronteira, 1997.

SIMBERLOFF, D. A. The proximate causes of extinction. In: RAUP, D. M.; JABLONSKI, D. (eds). Patterns and processes in the history of life. Berlin: Springer-Verlag, 1986. p. 259-276.

SIPINSKI, E. A. B. et al. Tendência populacional do papagaio-de-cara-roxa (Amazona brasiliensis) no litoral do estado do Paraná. Ornithologia, v. 6, n. 2, p. 136-143, 2014.

SOULÉ, M. E. Conservation biology: the science of scarcity and diversity. Sunderland: Sinauer Associates, 1996.

TRABULSI, L. R. Microbiologia. São Paulo: Ateneu, 1996. p. 386.

VAN DONGEN, W. F. D. et al. Age-related differences in the cloacal microbiota of a wild bird species. BMC Ecology, v. 13, p. 11, 2013.

XENOULIS, P. G. et al. Molecular characterization of the cloacal microbiota of wild and captive parrots. Veterinary Microbiology, v. 146, n. 3-4, p. 320-325, 2010. 